\title{
A comparison of the response of equine heart rate to different equine exercise regimes
}

\author{
C E S Barratt, J C Litten-Brown, C K Reynolds
}

University of Reading, Reading, United Kingdom

Email:c.e.barratt@reading.ac.uk

Introduction The regular monitoring of heart rate (HR) in response to exercise can be a beneficial practice in terms of improving equine training programs. It is know that equine HR increases linearly with workload (Harris et al., 2007) but the relationship between HR and exercise is usually determined using an equine treadmill, or a test completed on a track, access to which is often limited. Simple alternative methods which are highly reproducible and easy to complete could be of value. The aim of this study was to produce a simple method which compared the response of equine HR to different equine exercise regimes measured using an electronic monitor that is simple and easy to use. The simplicity of the method would mean that it can be completed by all equine owners/trainers and not limit equine cardiovascular research to only those who have access to an equine treadmill or outdoor track.

Materials and methods Five horses completed five different exercise regimes (TRT) in a replicated 5 X 5 Latin square design: TRT 1 - flatwork; TRT 2 - lungeing (work on a constant circle); TRT 3 - lungeing with a rider; TRT 4 - jumping in an indoor school and TRT 5 - riding outdoors in woodland. Each TRT lasted 30 minutes and consisted of ten minutes walk, ten minutes trot and ten minutes canter sequentially. Within each square horses completed successive exercise regimes over a period of two weeks with one or two days interval between completions of the TRT. The experiment was completed three times. Horses were of a similar size and fitness level and were offered the same basic diet of 6-8 $\mathrm{kg}$ concentrates daily and haylage ad libitum. One mare and four geldings were used. A polar HR monitoring system (Polar Vantage NV ${ }^{\mathrm{TM}}$, Polar Electro OY, Kempele, Finland), was used to measure HR from when the horse commenced at a walk and continued for the duration of each TRT. Recovery HR was recorded for ten minutes after exercise stopped as the horse was cooling down in walk. The monitoring system recorded HR every 15 seconds for the duration of each TRT and recovery period. Average HR was taken as an average of all HR recordings taken during the 30 minute TRT period. Basal HR was measured as an average of the first four HR recordings at the initiation of exercise. Statistical analysis was performed using Minitab (Version 15) using a General Linear Model testing effects of horse, square, period and TRT on average HR, basal HR, recovery HR (time taken for HR to decrease to basal HR level), time to reach maximum HR and maximum HR. Basal HR was used as a covariate for all responses except the basal HR mean values. When effects of TRT were significant a Tukey Pairwise Comparision test was used to determine differences between individual treatments.

Results Type of exercise has a significant effect on average and basal HR $(\mathrm{P}<0.001)$. There was no effect on the other variables. Individual horses had different average HR but the effect of horse was not significant for other variables.

Table 1 Effects of exercise regimes on average and basal HR (beats per minute (bpm)) in horses. (n = 15 per TRT; different superscripts show differences between individual treatments).

\begin{tabular}{|c|c|c|c|c|c|c|c|}
\hline & 1 & 2 & $\begin{array}{l}\text { Treatment } \\
3\end{array}$ & 4 & 5 & SEM & P-Value \\
\hline Average HR & $99.1^{\mathrm{ad}}$ & $87.0^{\mathrm{b}}$ & $96.2^{d}$ & $100.2^{\mathrm{a}}$ & $111.8^{\mathrm{c}}$ & 1.56 & 0.001 \\
\hline Basal HR & $36.8^{\mathrm{a}}$ & $36.6^{\mathrm{a}}$ & $37.3^{\mathrm{a}}$ & $41.4^{\mathrm{b}}$ & $41.4^{\mathrm{b}}$ & 1.57 & 0.001 \\
\hline
\end{tabular}

Conclusions An electronic HR monitor was a useful tool for monitoring HR response to exercise without specialist facilities. Of the exercise regimes compared, the highest average HR was measured during TRT 5 whilst TRT 1 was associated with the lowest. Basal HR was significantly higher during TRT 4 and 5, (table 1). Different ground surfaces, (Sloet van Oldruitenborgh-Osterbaan and Barnevald, 1995), different energy needs for different activities (Lawrence, 1997), or environmental factors (Harris et al., 2007) are a few possible reasons for the differences in average and basal HR measured. Effects of excitability cannot be ruled out; expectation may explain the higher basal HR for TRT 4 and 5 . Excitability can notably affect results when HR values are below 160 bpm (Harris et al., 2007). Horses 1 and 5 had significantly different HR $(\mathrm{P}<0.05)$ (data not shown). Although horses used were of similar fitness levels, a detailed assessment of fitness may be beneficial in future work. With advancement in technology available for equine cardiovascular research, such as the use of global positioning systems, (Harris et al., 2007), methods of assessing equine fitness and response to exercise will continue to improve and become more accessible for all members of the equine industry in the future.

Acknowledgements Dr. Darren Juniper, University of Reading, for advice and help on the completion of the statistical analysis

\section{References}

Harris, p. Marlin, D.J. Avidson, H. Rodgerson, J. Gregory, A. and Harison, D. 2007. Journal of Equine and Comparative Exercise Physiology 4, 15-21

Lawrence, M.L. 1997. Journal of Equine Veterinary Science 17, 368-369

Polar electro OY. 1995 Polar Vantage NV TM Users Manual, Fin 90440, Kemele, Finland

Sloet van Oldruitenborgh-Osterbaan, M. and Barnevald, A. 1995. Vet Record 137, 136-139 